Armin Saalmüller, Matthias J. Reddehase,

Hans-Jörg Bühring ${ }^{\nabla}$, Stipan Jonjic ${ }^{\circ}$ and

Ulrich H. Koszinowski

Federal Research Centre for Virus Diseases of Animals and Medical University Clinic ${ }^{\nabla}$, Tübingen

\section{Simultaneous expression of CD4 and CD8 antigens by a substantial proportion of resting porcine T lymphocytes*}

\begin{abstract}
The existence of four subpopulations in resting porcine T lymphocytes is documented. In addition to the two known subpopulations which are typified by a mutually exclusive expression of either the $\mathrm{CD} 8$ or the $\mathrm{CD} 4$ differentiation antigen, $\mathrm{CD} 4^{-} \mathrm{CD} 8^{+}$and $\mathrm{CD} 4^{+} \mathrm{CD} 8^{-} \mathrm{T}$ lymphocytes, respectively, two unusual subpopulations were prominent not only in peripheral blood, but also in lymphoid tissues: $\mathrm{CD} 4^{-} \mathrm{CD} 8^{-} \mathrm{T}$ lymphocytes expressing neither of these antigens and $\mathrm{CD} 4^{+} \mathrm{CD} 8^{+} \mathrm{T}$ lymphocytes coexpressing both antigens. While $\mathrm{CD} 4^{+} \mathrm{CD} 8^{+}$lymphoblasts have been found also in other species, resting $T$ lymphocytes with that phenotype are without precedent among all species analyzed to date. This unique composition of the porcine T lymphocyte population has to be taken into consideration when the swine is used as a large animal model in experimental medicine.
\end{abstract}

\section{Introduction}

The swine is important in experimental medicine by serving as a large animal model in transplantation surgery, in pharmacology, and for the study of pathogenesis in infectious diseases $[1,2]$.

Only recently were the porcine CD8 [3-5] and CD4 [3, 5] lymphocyte differentiation markers identified with monoclonal antibodies (mAb). Studies in several other species concurred in the conclusion that the expression of CD4 and CD8 cell surface glycoproteins is mutually exclusive in resting peripheral $\mathrm{T}$ lymphocytes and defines two functional subsets, MHC class II-restricted $\mathrm{T}$ lymphocytes predominantly associated with helper function and MHC class I-restricted T lymphocytes mainly performing cytolytic function, $T_{H}$ and $T_{C}$, respectively $[6,7]$. There is increasing evidence that $\mathrm{CD} 4$ and CD8 glycoproteins are directly involved as accessory molecules in the molecular complex formed during the recognition of antigens (reviewed in [8]). Simultaneous expression of both markers would therefore raise principal questions regarding restriction specificity and function.

Studying the phenotype of miniature swine peripheral blood T lymphocytes, Pescovitz, Lunney and Sachs noted the existence of a $\mathrm{CD}^{+} \mathrm{CD}^{+}$subpopulation [5]. Human T lymphocytes with that phenotype were found in a low proportion in peripheral blood, and were identified as activated, blast-like cells [9]. It could be demonstrated that $\mathrm{CD} 4^{+} \mathrm{CD} 8^{-}$human T lymphocytes express CD8 after in vitro activation [10] and, likewise, double expression by rat $\mathrm{T}$ lymphocytes was also linked to activation [11]. From these recent findings in other

[I 6225]

\footnotetext{
* This work was supported by the Deutsche Forschungsgemeinschaft, grant Ko 571/9-1.

- Present address: Department of Physiology and Immunology, Medical Faculty, University of Rijeka, 51000 Rijeka, Yugoslavia.
}

Correspondence: Matthias J. Reddehase, Federal Research Centre for Virus Diseases of Animals, P.O. Box 1149, D-7400 Tübingen, FRG

Abbreviations: FITC: Fluorescein isothiocyanate FMF: Flow microfluorometry mAb: Monoclonal antibody(ies) MLR: Mixed leukocyte reaction PE: Phycoerythrin species, it could be suspected that the presence of a porcine $\mathrm{CD} 4^{+} \mathrm{CD} 8^{+}$subset also just reflects activation. If that would be the case, the immune system of the swine would not be exceptional.

In a previous communication [12] we have defined a novel porcine $\mathrm{T}$ lymphocyte differentiation antigen expressed by resting $\mathrm{T}$ lymphocytes of either subset and down-regulated upon activation. This marker, referred to as $8 / 1$, could now be employed as an independent means for judging the activation state of $\mathrm{T}$ lymphocytes. We demonstrate that porcine $\mathrm{CD}^{+} \mathrm{CD}^{+}$cells constitute a major subset of $8 / 1^{+}$, i.e. resting $\mathrm{T}$ lymphocytes in peripheral blood and also in lymphoid tissues. In this respect the immune system of the swine is unique and contributes new information to basic immunology.

\section{Materials and methods}

\subsection{Cell separation procedures}

Mononuclear leukocytes were isolated by Lymphoprep (Nyegaard, Oslo, Norway) density gradient centrifugation of heparinized peripheral blood derived from adult (6-8-monthold) domestic swines, race Deutsches Landschwein. Suspensions of thymocytes, splenocytes and lymph node cells were purified accordingly. $T$ lymphocytes were enriched to a purity of $>98 \%$ by passage through nylon wool columns.

\subsection{Generation of lymphoblasts in the mixed leukocyte reaction (MLR)}

Nylon wool-purified peripheral blood T lymphocytes $\left(2 \times 10^{5} /\right.$ microculture) were cultivated for 8 days along with $2 \times 10^{5}$ 30-Gy $\gamma$-irradiated allogeneic mononuclear leukocytes [12].

\section{3 mAb and antisera}

The murine $\mathrm{mAb} 295 / 33$ (anti-CD8, $\operatorname{IgG}_{2 \mathrm{a}}, x$ ) and 8/1 (anti-8/1, $\left.\operatorname{IgG}_{2 \mathrm{~b}}, x\right)$ were established as described before $[4,12]$. The $\mathrm{mAb}$ 74-12-4 (anti-CD4, $\operatorname{IgG}_{2 \mathrm{~b}}, \boldsymbol{x}$ ) and 74-22-15 (anti-monocyte/granulocyte, $\left.\operatorname{IgG}_{1}, x\right)$, described by Pescovitz et al. [3], were obtained from the American Type Culture Collection 
(Rockville, MD). If not indicated otherwise, fluorescein isothiocyanate (FITC)-conjugated goat anti-mouse IgG $\mathrm{F}\left(\mathrm{ab}^{\prime}\right)_{2}$ (Jackson Laboratories, Avondale, PA) was used as second antibody for staining. $\mathrm{Ig}^{+}$cells were labeled with biotinylated goat anti-pig $\operatorname{IgG}(\mathrm{H}+\mathrm{L}$ chains; Vector Laboratories, Burlingame, CA) and FITC-conjugated streptavidin (Jackson Laboratories). Purification of $\mathrm{mAb}$ and conjugation with biotin was done as described before [12].

\subsection{Two-color flow microfluorometry (FMF)}

Two $\times 10^{6}-5 \times 10^{6}$ nylon wool-purified $\mathrm{T}$ lymphocytes were stained according to the following labeling sequence: (a) incubation with $\mathrm{mAb}$ anti-CD4; (b) staining with FITC-conjugated goat anti-mouse IgG $\mathrm{F}\left(\mathrm{ab}^{\prime}\right)_{2}$; (c) saturation of free binding sites of the FITC-conjugated $\mathrm{Ab}$ with nonlabeled mouse IgG (Jackson Laboratories); (d) incubation with biotinylated $\mathrm{mAb}$ anti-CD8; (e) staining with phycoerythrin (PE)-conjugated avidin (Becton Dickinson, Mountain View, CA). All incubations were done for $30 \mathrm{~min}$ on ice. The two-color fluorescence analysis was performed on a FACS IV (Becton Dickinson) essentially as described before [12].

Labeling and measurement artefacts were excluded as follows: (a) to compensate the overlap of the emission spectra of FITC and $\mathrm{PE}$, the amplifier was adjusted so that by analyzing a mixture of cells labeled with either FITC or PE, the fluorescence signals were orthogonal to each other and parallel to the respective axis in the two-color (PE, yellow and FITC, green) fluorescence display; (b) substitution of PE-conjugated avidin by Texas Red-conjugated avidin to ensure dye-independence of the fluorescence profiles; (c) label inversion to exclude staining artefacts.

Selective analysis of lymphocytes was achieved by electronic gating in the dual-parameter display of right-angle light scatter vs. forward light scatter. Relative log fluorescence intensities in the yellow (PE) and green (FITC) fluorescence axes were expressed on scales of 64 channels. Three-dimensional images of fluorescence profiles were obtained by connecting the cell frequency peaks of all net points in the $64 \times 64$ matrix (computer program provided by $M$. Blaurock, Medical University Clinic, Tübingen, FRG). In the two-dimensional plots, contour lines were set to represent $10,20,40,80,120$ and 160 cells per 50000 cells analyzed. Nonspecific staining with fluorescent conjugates alone served to discriminate between negative and positive signals.

\section{Results}

\subsection{Subpopulations in porcine thymus}

Staining of CD4 and CD8 molecules on porcine thymocytes led to the detection of four subpopulations by two-color FMF analysis (Fig. 1). A major subpopulation expressed both markers simultaneously (CD $4^{+} \mathrm{CD} 8^{+}, 59 \%$ in this individual), while minor subpopulations expressed either marker exclusively $\left(\mathrm{CD}^{-} \mathrm{CD}^{+}, 11 \%\right.$; and $\left.\mathrm{CD} 4^{+} \mathrm{CD} 8^{-}, 11 \%\right)$ or expressed neither $\left(\mathrm{CD}^{-} \mathrm{CD}^{-}, 19 \%\right)$. This marker distribution profile is similar to that found with the analogous murine CD4 (L3T4) and CD8 (Lyt-2) and human CD4 (T4) and CD8 (T8) markers in murine [13] and human [14] thymocytes, respectively.
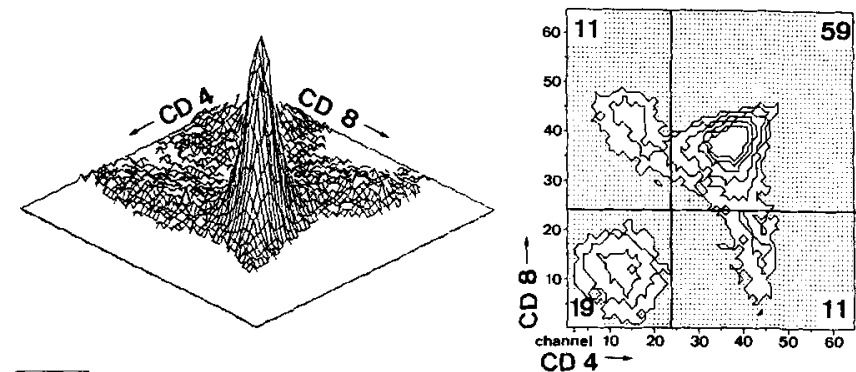

16225.1

Figure 1. Porcine thymocyte subpopulations defined by the expression of CD4 and CD8 antigens. After labeling with anti-CD4-FITC and anti-CD8-PE, $5 \times 10^{4}$ thymocytes were analyzed by two-color FMF. Data are displayed as a three-dimensional image (left) and as contour plot (right). Contour lines represent levels of 10, 20,40, 80, 120 and 160 cells. Double-negative cells are included in the quadrant below channel no. 24 (ordinate; CD8) and to the left of channel no. 24 (abscissa; CD4). The numbers in the corners represent the percentages of cells in the respective quadrants.
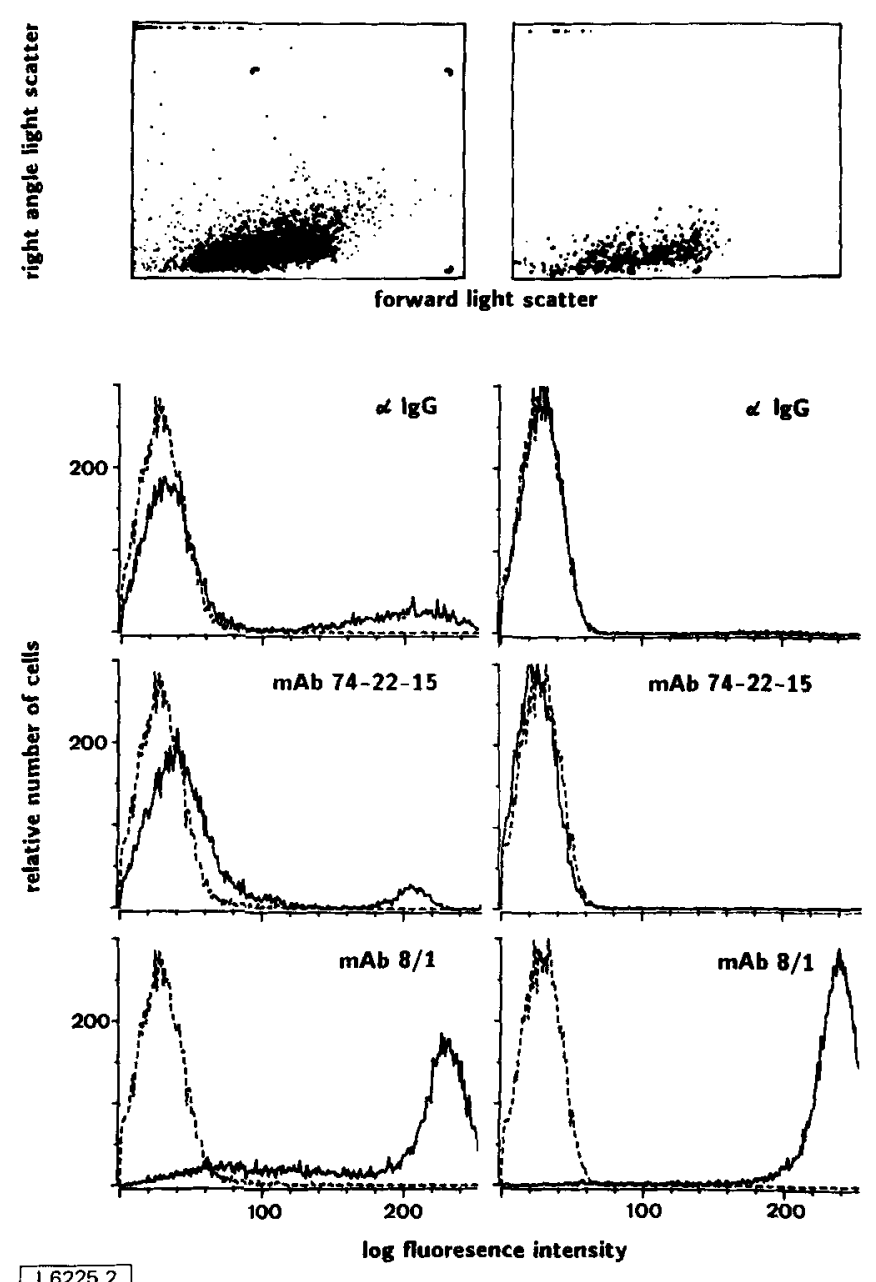

Figure 2. Purity and activation stage of T lymphocytes. Peripheral blood mononuclear cells were analyzed by single-parameter FMF before (left column; electronic gate indicated by angles in the scatter plot) or after nylon wool purification and gating on resting lymphocytes (right column). Cells were stained with anti-IgG (second row) or with $m A b$ 74-22-15 (third row) or with $m A b$ 8/1 (fourth row). Relative fluorescence intensities are expressed on a $\log$ scale with $10^{4}$ cells analyzed (solid graphs). Labeling with the respective FITC conjugates provided the negative controls (dashed graphs). 


\subsection{Selective analysis of resting peripheral blood $T$ lymphocytes}

Nylon wool purification and electronic gating were combined to achieve the selective analysis of resting $\mathrm{T}$ lymphocytes (Fig. 2). The scatter plots (first row) show the composition of the cell population before (left) and after (right) nylon wool purification with regard to physical properties defined by forward and right-angle light scatter. After purification, the electronic gate was set to exclude monocytes and lymphoblasts from the analysis. Control of the purity by single-color FMF (compare left and right column) demonstrated the depletion of Ig-bearing B lymphocytes (second row), and also the exclusion of monocytes (third row). All cells expressed the 8/1 antigen (fourth row). Thus, lymphocytes that entered the two-color FMF analysis were characterized as resting $T$ lymphocytes by physical and serological criteria.

\subsection{Four subpopulations of resting porcine peripheral blood $T$ lymphocytes}

Resting $\mathrm{T}$ lymphocytes were purified as outlined in Fig. 2 and were then subjected to two-color FMF analysis for the detection of CD4 and CD8 antigens (Fig. 3). Four subpopulations could be discriminated in porcine $\mathrm{T}$ lymphocytes. In addition to the expected subpopulations $\mathrm{CD}^{-} \mathrm{CD}^{+}(30 \%$ in this individual) and $\mathrm{CD}^{+} \mathrm{CD}^{-}(12 \%)$, double-negative $\mathrm{CD} 4^{-} \mathrm{CD} 8^{-}$ $(15 \%)$ and double-positive $\mathrm{CD} 4^{+} \mathrm{CD} 8^{+}(43 \%)$ subpopulations were prominent. The $\mathrm{CD} 4^{-} \mathrm{CD}^{+}$subpopulation could be further subdivided by means of low (peak intensity at ordinate channel no. 38) and high (peak intensity at ordinate channell no. 55) expression of the CD8 antigen. In the double-positive subset, the expression of CD8 (peak intensity at ordinate channel no. 37) corresponded to that of the $\mathrm{CD} 4^{-} \mathrm{CD} 8^{+}$low subpopulation.

By collecting a random sample of 12 outbred individuals, the existence of four porcine $T$ lymphocyte subpopulations could be confirmed (Fig. 4). The variance in the proportions was pronounced in the double-positive (8-64\%) and double-negative $(2-52 \%)$ subsets and appeared to be inversely related, while the proportion of $\mathrm{CD} 4^{-} \mathrm{CD} 8^{+} \mathrm{T}$ lymphocytes was almost constant (21-39\%).

\subsection{Demonstration of four $T$ lymphocyte subpopulations in lymphoid tissues}

To test whether double-negative and double-positive lymphocytes also reside in lymphoid tissues, nylon wool-purified $\mathrm{T}$

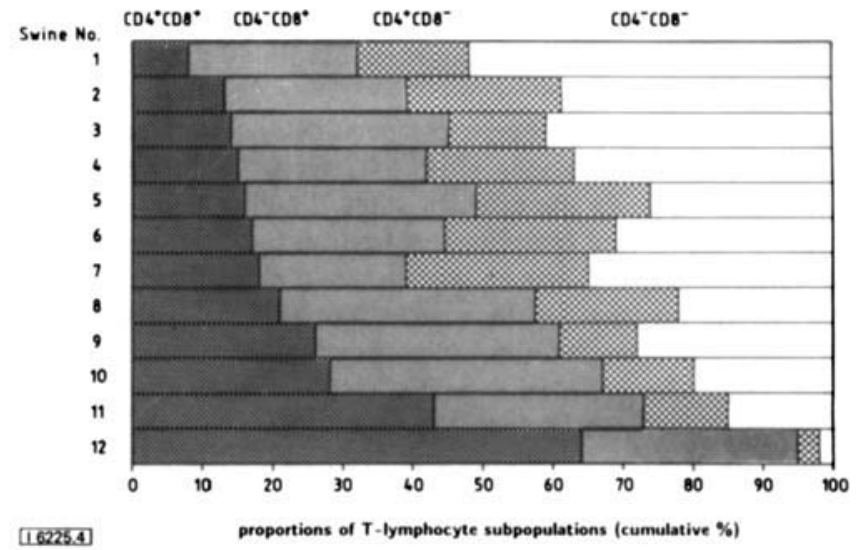

Figure 4. Variation in the proportions of $T$ lymphocyte subpopulations. Peripheral blood $\mathrm{T}$ lymphocytes derived from 12 swines, randomly selected during a period of 2 years, were purified as detained in Fig. 2 and analyzed for the expression of $\mathrm{CD} 4$ and $\mathrm{CD} 8$ antigens as exemplified in Fig. 3 for swine no. 11. The individuals were signified with rank numbers according to increasing percentages of doublepositive $\mathrm{T}$ lymphocytes.

lymphocytes derived from spleen, mesenteric lymph nodes and tonsils were subjected to two-color FMF analysis (Fig. 5). In essence, all four subpopulations known from the analysis of peripheral blood $\mathrm{T}$ lymphocytes were also detectable in lymphoid tissues. Specifically, in this individual (swine no. 3), double-negative cells were most frequent in spleen $(63 \%)$, while the classical subsets $\mathrm{CD} 4^{-} \mathrm{CD}^{+}(25 \%)$ and $\mathrm{CD} 4^{+} \mathrm{CD} 8^{-}$ $(39 \%)$ dominated the mesenteric lymph nodes, but were almost absent (14\% and 6\%, respectively) from tonsils.

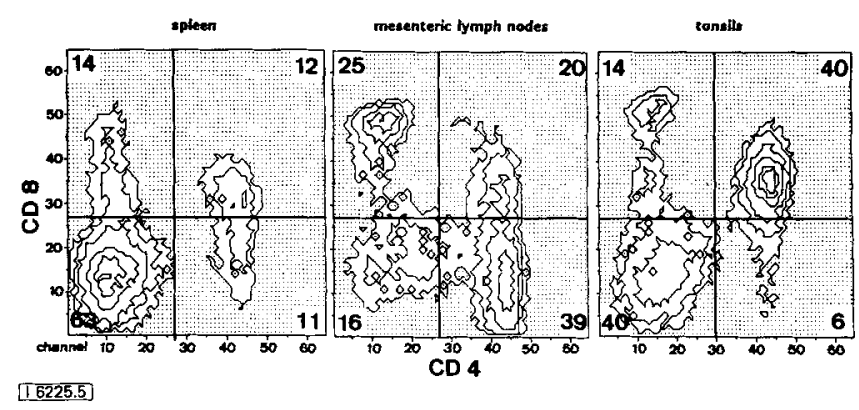

Figure 5. Proportions of subpopulations in $\mathrm{T}$ lymphocytes derived from different lymphoid tissues. Fifty thousand nylon wool-purified $\mathrm{T}$ lymphocytes from spleen, mesenteric lymph nodes and tonsils of swine no. 3 were analyzed.

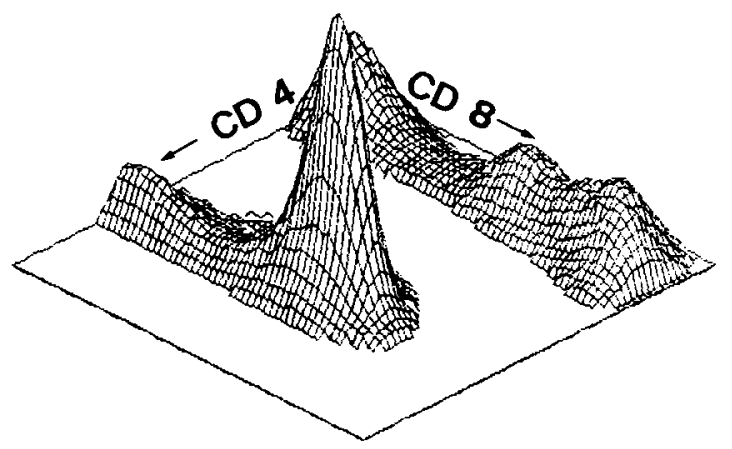

16225.3

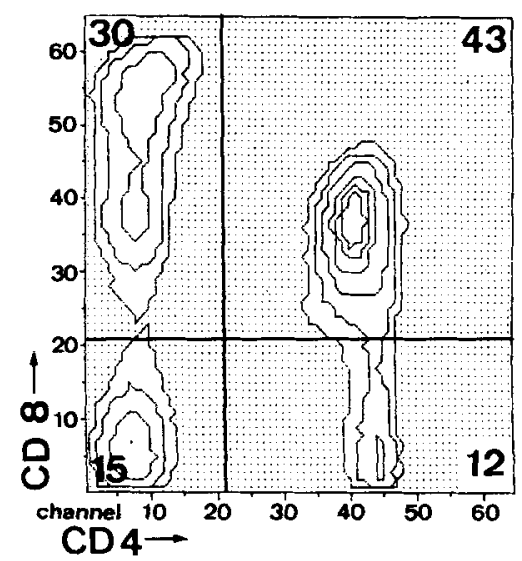

Figure 3. Subpopulations of resting peripheral blood $\mathrm{T}$ lymphocytes defined by the expression of $\mathrm{CD} 4$ and CD8 antigens. The two-color FMF analysis was performed with $1 \times 10^{6}$ $8 / 1^{+}$, i.e. resting $T$ lymphocytes purified as detailed in Fig. 2. Contour lines represent levels of 200 , $400,800,1600,2400$ and 3200 cells. 


\subsection{Accumulation of lymphoblasts with the phenotype $\mathrm{CD4}^{+} \mathrm{CDB}^{+}$during an allogeneic MLR}

To determine the phenotype of specifically sensitized $\mathrm{T}$ lymphoblasts, $T$ lymphocytes (Fig. 6A) were stimulated in an allogeneic MLR. In the effector stage the electronic gate was either set on resting lymphocytes that had not participated in the reaction (Fig. 6B) or on lymphoblasts characterized physically by the position in the scatter plot (Fig. 6C). Two-color FMF analysis revealed similar proportions of subpopulations in the responder population before stimulation and in the resting fraction of the MLR (compare A and B), while the effector population (C) was composed of two subpopulations: $\mathrm{CD} 4^{-} \mathrm{CD} 8^{+}$lymphoblasts with low or high density of the CD8 antigen (together 46\%) and double-positive lymphoblasts $(40 \%)$.

Double-positive lymphocytes positively selected by FACS sorting (A. Saalmüller et al., to be published) could be stimulated with concanavalin $\mathrm{A}$ and then further propagated in the presence of recombinant interleukin 2 without change in the phenotype. This demonstrated that the resting $\mathrm{CD} 4^{+} \mathrm{CD}^{+}$ subset is not refractory to activation and that the coexpression of porcine CD4 and CD8 antigens is not transient.
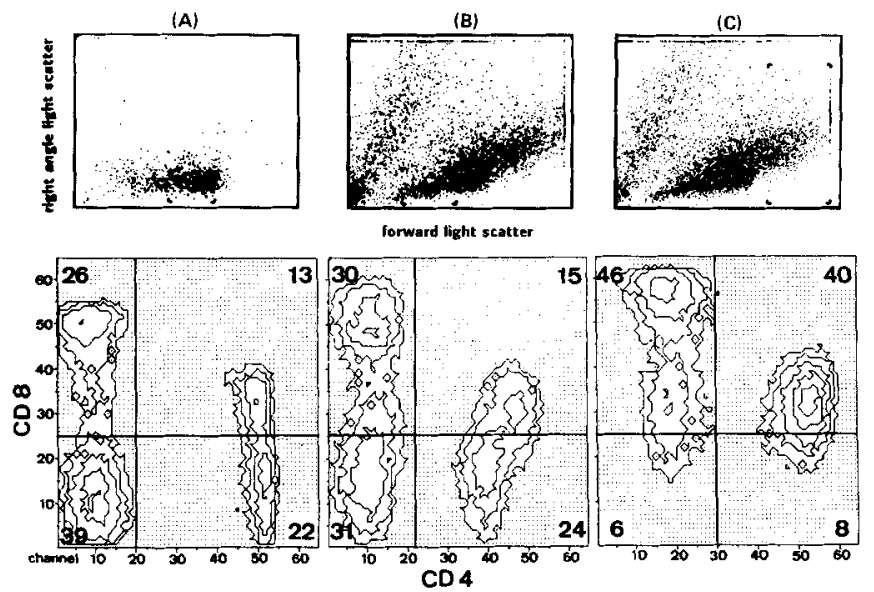

$1 \overline{6225.6}$

Figure 6. Coexpression of CD4 and CD8 antigens on activated T lymphocytes. Two-color FMF analysis of nylon wool-purified peripheral blood $\mathrm{T}$ lymphocytes of swine no. 2 before $(\mathrm{A})$ and after in vitro activation in the MLR $(B, C)$. The angles in the scatter plots demarkate the electronic gate, which was set on resting lymphocytes in (A) and (B), and on lymphoblasts in (C). Each analysis was performed with 50000 cells.

\section{Discussion}

Our study has revealed the regular presence of the unusual phenotypes $\mathrm{CD} 4^{-} \mathrm{CD} 8^{-}$and $\mathrm{CD} 4^{+} \mathrm{CD} 8^{+}$among resting porcine $\mathrm{T}$ lymphocytes.

It has been proposed that during thymic ontogeny $\mathrm{CD}^{-} \mathrm{CD} 8^{-}$ precursor cells differentiate into $\mathrm{CD} 4^{+} \mathrm{CD}^{+}$thymocytes, which, upon further differentiation, lose either CD4 or CD8 giving rise to the mature phenotypes $\mathrm{CD} 4^{-} \mathrm{CD} 8^{+}$and $\mathrm{CD} 4^{+} \mathrm{CD} 8^{-}[6,15]$. In the mouse, an extrathymic presence of double-negative $T$ lymphocytes appears to be associated with defective differentiation $[16,17]$. Lanier and colleagues [18] have shown that up to $23 \%$ of human blood lymphocytes derived from normal donors can comprise $\mathrm{CD} 3^{+} \mathrm{CD} 4^{-} \mathrm{CD} 8^{-} \mathrm{T}$ lymphocytes. In sheep T lymphocytes, Mackay et al. [19] identified a third subpopulation, which is characterized as $\mathrm{CD} 4^{-} \mathrm{CD} 8^{-}$and positive for the expression of a novel differentiation antigen, referred to as SBU-T19. This subpopulation makes up 6-29\% of peripheral blood lymphocytes, but only $1-6 \%$ of lymph node lymphocytes, interpreted as being in transit through the node rather than resident. In this respect, the findings in sheep markedly differ from the situation in swine, where double-negative lymphocytes are a prominent population in spleen, mesenteric lymph nodes and tonsils. Another difference between the immune systems of swine and sheep is the absence of a $\mathrm{CD} 4^{+} \mathrm{CD} 8^{+}$subpopulation in sheep T lymphocytes [19].

In human peripheral blood $\mathrm{T}$ lymphocytes, Blue and colleagues detected $0.5-8 \% \mathrm{CD}^{+} \mathrm{CD}^{+}$lymphoblasts [9]. This finding suggested that the $6-15 \%$ of $\mathrm{CD}^{+} \mathrm{CD}^{+} \mathrm{T}$ lymphocytes detected previously in the peripheral blood of miniature swines [5] may also have been lymphoblasts. We have taken care to discriminate clearly between resting and activated lymphocytes by employing scatter plot position and expression of the differentiation marker $8 / 1$ [12] as criteria. Our data add to previous knowledge the important information that porcine $\mathrm{CD} 4$ and $\mathrm{CD} 8$ antigens are regularly coexpressed on a significant proportion $(8-64 \%)$ of the small, resting peripheral $\mathrm{T}$ lymphocytes.

So far, we and other investigators have not been able to ascribe a specific function to this unusual subpopulation. In murine $\mathrm{CD} 4^{+} \mathrm{CD} 8{ }^{+}$clones, the $\mathrm{CD} 8$ molecules were found to be expressed as Lyt-2 homodimers, which did not participate in antigen recognition that was class II restricted and involved only the CD4 molecule [20]. It is questionable, however, whether these rare murine clones are representative of doubleexpressing $\mathrm{T}$ lymphocytes in general.

The genealogy of the porcine peripheral $\mathrm{CD}^{+} \mathrm{CD}^{+} \mathrm{T}$ lymphocytes is unknown. Two alternatives can be considered. First, these cells could represent immature precursors emigrated from the thymus without prior differentiation. Second, these cells could represent memory $\mathrm{T}$ helper lymphocytes that have acquired the CD8 antigen upon prior sensitization, but did not lose CD8 after reversion to small lymphocytes. Sorting of double-expressing $T$ lymphocytes derived from specifically primed swines followed by specific in vitro restimulation assays should enable differentiation between these alternatives.

We appreciate the help of Dr. V. Ohlinger and Dr. G. Hess in veterinary surgery, the technical assistance of Anke Lüske and Irene Huber, and the secretarial assistance of Sabine Grau.

Received June 5, 1987; in revised form July 10, 1987.

\section{References}

1 Tumbleson, M. E. (Ed.), Swine in Biomedical Research, Plenum Press, New York 1986.

2 Bielefeldt Ohmann, H. and Babiuk, L. A., J. Gen. Virol. 1986. 66: 1.

3 Pescovitz, M. D., Lunney, J. K. and Sachs, D. H., J. Immunol. 1984. 133: 368 
4 Jonjić, S. and Koszinowski, U. H., J. Immunol. 1984. 133: 647.

5 Pescovitz, M. D., Lunney, J. K. and Sachs, D. H., J. Immunol. 1985. 134: 37.

6 Reinherz, E. and Schlossman, S., Cell 1980. 19: 821.

7 Swain, S. I., Immunol. Rev. 1983. 74: 129.

8 Goverman, J., Hunkapiller, T. and Hood, L., Cell 1986. 45: 475.

9 Blue, M.-L., Daley, J. F., Levine, H. and Schlossman, S. F., J. Immunol. 1985. 134: 2281.

10 Blue, M.-L., Daley, J. F., Levine, H., Craig, K. A. and Schlossman, S. F., J. Immunol. 1986. 137: 1202.

11 Bevan, D. J. and Chisholm, P. M., Immunology 1986. 59: 621.

12 Saalmüller, A., Jonjić, S., Bühring, H.-J., Reddehase, M. J. and Koszinowski, U. H., J. Immunol. 1987. 138: 1852.

13 Scollay, R., Bartlett, P. and Shortman, K., Immunol. Rev. 1984. 82: 79.
14 Lanier, L. L., Allison, J. P. and Phillips, J. H., J. Immunol. 1986. 137: 2501.

15 Reinherz, E. L., Kung, P. C., Goldstein, G., Levey, R. H. and Schlossman, S. F., Proc. Natl. Acad. Sci. USA 1980. 77: 1588.

16 Davignon, J.-L., Budd, R. C., Ceredig, R., Piguet, P. F., MacDonald, H. R., Cerottini, J.-C., Vassalli, P. and Izui, S., J. Immunol. 1985. 135: 2423.

17 MacDonald, H. R., Blanc, C., Lees, R. K. and Sordat, B., J. Immunol. 1986. 136: 4337.

18 Lanier, L. L., Ruitenberg, J. J. and Phillips, J. H., J. Exp. Med. 1986. 164: 339.

19 Mackay, C. R., Maddox, J. F. and Brandon, M. R., Eur. J. Immunol. 1986. 16: 19.

20 Fazekas de St. Groth, B., Gallagher, P. F. and Miller, J. F. A. P., Proc. Natl. Acad. Sci. USA 1986. 83: 2594. 